\title{
MAXIMAL ATTRACTOR FOR THE SYSTEM OF ONE-DIMENSIONAL POLYTROPIC VISCOUS IDEAL GAS
}

BY

SONGMU ZHENG (Institute of Mathematics, Fudan University, Shanghai 200433, People's Republic of China)

AND

YUMING QIN (Department of Mathematics, Henan University, Kaifeng 475001, People's Republic of China)

Abstract. In this paper, the dynamics for the system of polytropic viscous ideal gas is investigated. One of the important features of this problem is that the metric spaces $H^{(1)}$ and $H^{(2)}$ that we work with are two incomplete metric spaces, as can be seen from the constraints $\theta>0$ and $u>0$ with $\theta$ and $u$ begin absolute temperature and specific volume, respectively. For any constants $\beta_{1}, \beta_{2}, \beta_{3}, \beta_{4}, \beta_{5}$ satisfying certain conditions, two sequences of closed subspaces $H_{\beta}^{(i)} \subset H^{(i)}(i=1,2)$ are found, and the existence of two maximal (universal) attractors in $H_{\beta}^{(1)}$ and $H_{\beta}^{(2)}$ is proved.

1. Introduction. This paper is concerned with existence of maximal (universal) attractors for the system of one-dimensional polytropic viscous ideal gas. The referential (Lagrangian) form of the conservation laws of mass, momentum, and energy for this kind of gas with the reference density $\rho_{0}=1$ can be written as follows (see [8]):

$$
\begin{aligned}
u_{t}-v_{x} & =0, \\
v_{t}+\left(\frac{R \theta}{u}\right)_{x}-\left(\frac{\mu v_{x}}{u}\right)_{x} & =0, \\
C_{V} \theta_{t}+\frac{R \theta v_{x}}{v}-\left(\frac{K \theta_{x}}{u}\right)_{x}-\frac{\mu v_{x}^{2}}{u} & =0 .
\end{aligned}
$$

Here subscripts denote partial differentiations, and $u, v, \theta$ denote the specific volume, velocity and absolute temperature, respectively. $R, \mu, C_{V}$ and $K$ are given positive constants. We consider problem (1.1)-(1.3) in the region $\{0 \leq x \leq 1, t \geq 0\}$ subject to the

Received February 21, 2000.

2000 Mathematics Subject Classification. Primary 35Q72, 35B30, 35B40.

Key words and phrases. One-dimensional polytropic viscous ideal gas, incomplete metric space, absorbing set, maximal attractor, universal attractor.

E-mail address: szheng@fudan.ac.cn

E-mail address: qinyuming@mail.henu.edu.cn

(C)2001 Brown University 
boundary conditions

$$
v(0, t)=v(1, t)=0, \quad \theta_{x}(0, t)=\theta_{x}(1, t)=0,
$$

and the initial conditions

$$
u(x, 0)=u_{0}(x), \quad v(x, 0)=v_{0}(x), \quad \theta(x, 0)=\theta_{0}(x) \quad \text { on }[0,1] .
$$

First, let us recall that the results on global existence, uniqueness and asymptotic behaviour of solutions to problem (1.1)-(1.5) have been established in [8], [11]; see also [7], [5], [1] and the references cited there.

In this paper, we would like to further study the dynamics of problem (1.1)-(1.5), namely, we want to prove the existence of maximal (universal) attractors. Before stating and proving our results, let us first explain some mathematical difficulties in studying the dynamics of this problem. First, from physical reasons, the special volume $u$ and the absolute temperature $\theta$ must be positive for all time. These constraints give rise to some severe mathematical difficulties. For instance, we must work on incomplete metric spaces $H^{(1)}$ and $H^{(2)}, H^{(2)} \subset H^{(1)}$, with these constraints. The nonlinear semigroup $S(t)$ defined by problem (1.1)-(1.5) maps each $H^{(1)}$ and $H^{(2)}$ into itself. It is clear from equations (1.2)-(1.3) that we cannot continuously extend the semigroup $S(t)$ to the closure of $H^{(1)}$ and $H^{(2)}$. Notice the following significant differences between the study of global existence and the study of existence of a maximal attractor: for the study of global existence, the initial datum is given while for the study of existence of a maximal attractor in a certain metric space, the initial data are varying in that space. Since the maximal attractor is just the $\omega$-limit set of an absorbing set, the requirement on completeness of spaces is needed. To overcome this severe mathematical difficulty, we restrict ourselves to a sequence of closed subspaces of $H^{(1)}$ and $H^{(2)}$ (see the definition below). It turns out that it is very crucial to prove that the orbit starting from any bounded set of this closed subspace will reenter this subspace and stay there after a finite time, which should be uniform with respect to all orbits starting from a bounded set; otherwise, there is no grounds for talking about the existence of an absorbing set and maximal attractor in this subspace. The proof of this fact becomes an essential part of our paper. Secondly, two quantities, i.e., the total mass and energy, are conserved. Indeed, if we integrate the first equation (1.1) with respect to $x$ and $t$ and exploit the boundary conditions, we will end up with

$$
\int_{0}^{1} u(x, t) d x=\int_{0}^{1} u_{0}(x) d x, \quad \forall t>0 .
$$

Next, if we multiply (1.2) by $v$, and integrate the resultant and also the third equation with respect to $x$ and $t$, then add together, we finally get

$$
\int_{0}^{1}\left(C_{V} \theta+\frac{v^{2}}{2}\right) d x=\int_{0}^{1}\left(C_{V} \theta_{0}(x)+\frac{v_{0}^{2}}{2}(x)\right) d x .
$$

These two conservations indicate that there can be no absorbing set for initial data varying in the whole space. Instead, we should rather consider the dynamics in a sequence of closed subspaces defined by some parameters. In this regard, the situation is quite similar to that encountered for the single Cahn-Hilliard equation in the isothermal case (see [18]) and the coupled Cahn-Hilliard equations (see [14]). Therefore, one of the 
key issues in the present paper is how to choose these closed subspaces. Finally, (1.1)(1.3) is a hyperbolic-parabolic coupled system. It turns out that in general the orbit is not compact. In order to prove the existence of a maximal attractor by the theory presented in [18] by Temam, we have either to show the uniform compactness of the orbit of semigroup $S(t)$ for large time or to show that we can decompose $S(t)$ into two parts, $S_{1}(t)$ and $S_{2}(t)$, with $S_{1}$ being uniformly compact for large time and $S_{2}$ going to zero uniformly. Since equations (1.1)-(1.3) is a hyperbolic-parabolic coupled system, the orbit is not compact. Moreover, since our system is quasilinear, the usual method of decomposition into two parts for a semilinear system (see [4]) does not seem feasible. To overcome this difficulty, we will adopt an approach motivated by the idea in [2].

From now on, without loss of generality we always assume that $C_{V}=R=\mu=K=1$. Now let us consider the spaces

$$
\begin{array}{r}
H^{(1)}=\left\{(u, v, \theta) \in H^{1}[0,1] \times H^{1}[0,1] \times H^{1}[0,1]: u(x)>0, \theta(x)>0, x \in[0,1]\right. \\
\left.\left.v\right|_{x=0}=\left.v\right|_{x=1}=0\right\}
\end{array}
$$

and

$$
\begin{aligned}
& H^{(2)}=\left\{(u, v, \theta) \in H^{2}[0,1] \times H^{2}[0,1] \times H^{2}[0,1]: u(x)>0, \theta(x)>0, x \in[0,1]\right. \\
&\left.\left.v\right|_{x=0}=\left.v\right|_{x=1}=\left.\theta_{x}\right|_{x=0}=\left.\theta_{x}\right|_{x=1}=0\right\}
\end{aligned}
$$

which become two metric spaces when equipped with the metrics induced from the usual norms. In the above, $H^{1}, H^{2}$ are the usual Sobolev spaces.

Let $\beta_{i}(i=1, \ldots, 5)$ be any given constants such that $\beta_{1} \in \mathbb{R}, \beta_{2}>0, \beta_{4} \geq \frac{e^{\beta_{1}}}{\beta_{2}}>$ $\beta_{3}>0,0<\beta_{5}<\beta_{2}$ are arbitrarily given constants, and let

$$
\begin{array}{r}
H_{\beta}^{(i)}:=\left\{(u, v, \theta) \in H^{(i)}: \int_{0}^{1}(\ln (\theta)+\ln (u)) d x \geq \beta_{1}, \beta_{5} \leq \int_{0}^{1}\left(\theta+v^{2} / 2\right) d x \leq \beta_{2},\right. \\
\left.\beta_{3} \leq \int_{0}^{1} u d x \leq \beta_{4}, \beta_{5} / 2 \leq \theta \leq 2 \beta_{2}, \beta_{3} / 2 \leq u \leq 2 \beta_{4}\right\}, \quad i=1,2 .
\end{array}
$$

Clearly, $H_{\beta}^{(i)}$ is a sequence of closed subspaces of $H^{(i)}(i=1,2)$. Later on we will show that the first three constraints are invariant. However, the last two constraints are not invariant. These two constraints are just introduced to overcome the difficulty that the original spaces are incomplete. As mentioned before, it is very crucial to prove that the orbit starting from any bounded set of $H_{\beta}^{(i)}$ will reenter $H_{\beta}^{(i)}$ after a finite time.

We are now in a position to state our main theorem.

THEOREM 1.1. The nonlinear semigroup $S(t)$ defined by the solution to problem (1.1)(1.5) maps $H^{(i)}(i=1,2)$ into itself. Moreover, for any $\beta_{i}(i=1, \ldots, 5)$ with $\beta_{1}<$ $0, \beta_{2}>0, \beta_{4} \geq \frac{e^{\beta_{1}}}{\beta_{2}}>\beta_{3}>0,0<\beta_{5}<\beta_{2}$, it possesses in $H_{\beta}^{(i)}$ a maximal attractor $\mathcal{A}_{i, \beta}(i=1,2)$.

REMARK 1.1. The set $\mathcal{A}_{i}=\bigcup_{\beta_{1}, \beta_{2}, \beta_{3}, \beta_{4}, \beta_{5}} \mathcal{A}_{i, \beta}(i=1,2)$ is a global noncompact attractor in the metric space $H^{(i)}$ in the following sense that it attracts any bounded sets of $H^{(i)}$ with constraints $u \geq \eta_{1}, \theta \geq \eta_{2}$ with $\eta_{1}, \eta_{2}$ being any given positive constants. 
The notation in this paper will be as follows: $L^{p}, 1 \leq p \leq+\infty, W^{m, p}, m \in N, H^{1}=$ $W^{1,2}, H_{0}^{1}=W_{0}^{1,2}$ denote the usual (Sobolev) spaces on $(0,1)$. In addition (,) stands for the inner product in $L^{2}$, and $\|\cdot\|_{B}$ denotes the norm in the space $B$; we also put $\|\cdot\|=\|\cdot\|_{L^{2}}$. We denote by $C^{k}(I, B), k \in N_{0}$, the space of $k$-times continuously differentiable functions from $I \subseteq R$ into a Banach space $B$, and likewise by $L^{p}(I, B)$, $1 \leq p \leq+\infty$ the corresponding Lebesgue spaces. Subscripts $t$ and $x$ denote the (partial) derivatives with respect to $t$ and $x$, respectively. We use $C_{0}^{(i)}, i=1,2$, to denote the universal constant depending only on the $H^{(i)}$ norm of initial data and $\min _{x \in[0,1]} u_{0}(x)$. $C_{\beta}$ denotes the universal constant depending only on $\beta_{i}(i=1, \ldots, 5)$ but independent of initial data. $C_{\beta}^{(i)}$ denotes the universal constant depending on $\beta_{j}(j=1,2,3,4,5)$, the $H^{(i)}$ norm of initial data, and $\min _{x \in[0,1]} u_{0}(x)$.

2. Nonlinear semigroup on $H^{(2)}$. As mentioned in the previous section, for any initial data $\left(u_{0}, v_{0}, \theta_{0}\right) \in H^{(1)}$, the results on global existence, uniqueness and asymptotic behaviour of solutions to problem (1.1)-(1.5) have been established in [8], [11]. More precisely, we have

LEMma 2.1. $S(t)$ defines a nonlinear $C_{0}$-semigroup on $H^{(1)}$ such that for any $\left(u_{0}, v_{0}, \theta_{0}\right)$ $\in H^{(1)}, S(t)\left(u_{0}, v_{0}, \theta_{0}\right)=(u(t), v(t), \theta(t)) \in C\left([0,+\infty) ; H^{(1)}\right), u_{t}, \theta_{x}, v_{x}, u_{x}, v_{x x}, \theta_{x x} \in$ $L^{2}\left([0,+\infty) ; L^{2}\right)$. Moreover,

$$
\begin{gathered}
0<\theta(x, t) \leq C_{0}^{(1)} \text { on }[0,1] \times[0, \infty), \\
0<1 / C_{0}^{(1)} \leq u(x, t) \leq C_{0}^{(1)} \text { on }[0,1] \times[0, \infty), \\
\|u(t)\|_{H^{1}}^{2}+\|\theta(t)\|_{H^{1}}^{2}+\|v(t)\|_{H^{1}}^{2}+\int_{0}^{t}\left(\left\|u_{x}\right\|^{2}+\left\|v_{x}\right\|^{2}\right. \\
\left.+\left\|\theta_{x}\right\|^{2}+\left\|v_{x x}\right\|^{2}+\left\|\theta_{x x}\right\|^{2}\right)(\tau) d \tau \leq C_{0}^{(1)}, \quad \forall t>0 . \\
\left\|\left(u(t)-\overline{u_{0}}, v(t), \theta(t)-\bar{\theta}\right)\right\|_{H^{1}} \rightarrow 0, \quad \text { as } t \rightarrow+\infty,
\end{gathered}
$$

where

$$
\overline{u_{0}}=\int_{0}^{1} u_{0}(x) d x, \quad \bar{\theta}=\int_{0}^{1}\left(v_{0}^{2}(x) / 2+\theta_{0}(x)\right) d x .
$$

Now we have

THEOREM 2.1. $S(t)$ defines a nonlinear $C_{0}$-semigroup on $H^{(2)}$.

Proof. The proof of Theorem 2.1 is divided into a series of lemmas.

LEMmA 2.2. For each $t \geq 0$, there exists a point $x_{1}=x_{1}(t) \in[0,1]$ such that the solution $u(x, t)$ to problem $(1.1)-(1.5)$ has the following expression:

$$
u(x, t)=D(x, t) Z(t)\left(1+\int_{0}^{t} \frac{\theta(x, \tau)}{D(x, \tau) Z(\tau)} d \tau\right)
$$


where

$$
\begin{aligned}
D(x, t)= & u_{0}(x) \exp (B(x, t)) \\
B(x, t)= & \int_{x_{1}(t)}^{x} v(y, t) d y-\int_{0}^{x} v_{0}(y) d y \\
& +\left(\int_{0}^{1} u_{0}(x) d x\right)^{-1} \int_{0}^{1} u_{0}(x) \int_{0}^{x} v_{0}(y) d y d x \\
Z(t)= & \exp \left(-\left(\int_{0}^{1} u_{0}(x) d x\right)^{-1} \int_{0}^{t} \int_{0}^{1}\left(v^{2}+\theta\right)(x, \tau) d x d \tau\right) .
\end{aligned}
$$

Proof. See, e.g., [12].

LEMma 2.3. For any $\left(u_{0}, v_{0}, \theta_{0}\right) \in H^{(2)}$, the unique global solution $(u, v, \theta)$ of problem (1.1)-(1.5) belongs to $C\left([0,+\infty) ; H^{(2)}\right)$. Moreover,

$$
\|(u(t), v(t), \theta(t))\|^{H^{(2)}} \leq C_{0}^{(2)}, \quad \forall t>0 .
$$

Proof. By the dense argument, it suffices to prove $(2.10)$ when $\left(u_{0}, v_{0}, \theta_{0}\right)$ belongs to $H^{(2)}$ and is smooth enough. By the results in [11], we know that there exists a unique smooth solution $(u(t), v(t), \theta(t))$ to problem (1.1)-(1.5). By equation (1.3), Lemma 2.1, and Sobolev's imbedding theorem, we have

$$
\begin{aligned}
& \int_{0}^{t}\left\|\theta_{t}\right\|^{2}(\tau) d \tau \\
& \quad \leq C_{0}^{(1)} \int_{0}^{t}\left(\left\|v_{x}\right\|^{2}+\left\|\theta_{x x}\right\|^{2}+\left\|\theta_{x}\right\|\left\|\theta_{x x}\right\|\left\|u_{x}\right\|^{2}+\left\|v_{x}\right\|^{3}\left\|v_{x x}\right\|\right)(\tau) d \tau \\
& \quad \leq C_{0}^{(1)} \int_{0}^{t}\left(\left\|v_{x}\right\|^{2}+\left\|v_{x x}\right\|^{2}+\left\|\theta_{x}\right\|^{2}+\left\|\theta_{x x}\right\|^{2}\right)(\tau) d \tau \\
& \quad \leq C_{0}^{(1)}
\end{aligned}
$$

Differentiating (2.1) with respect to $t$, then multiplying the resultant by $v_{t}$ and integrating over $(0,1)$, we get

$$
\begin{aligned}
\frac{d}{d t}\left\|v_{t}\right\|^{2} & +\frac{1}{C_{0}^{(1)}}\left\|v_{x t}\right\|^{2} \\
& \leq C_{0}^{(1)}\left(\left\|\theta_{t}\right\|+\left\|v_{x}\right\|+\left\|v_{x}^{2}\right\|\right)\left\|v_{x t}\right\| \\
& \leq \frac{1}{2 C_{0}^{(1)}}\left\|v_{x t}\right\|^{2}+C_{0}^{(1)}\left(\left\|\theta_{t}\right\|^{2}+\left\|v_{x}\right\|^{2}+\left\|v_{x}\right\|^{3}\left\|v_{x x}\right\|\right) .
\end{aligned}
$$

Then integrating with respect to $t$ and applying Lemma 2.1 yields

$$
\left\|v_{t}(t)\right\|^{2} \leq C_{0}^{(2)}, \quad \int_{0}^{t}\left\|v_{x t}\right\|^{2} d \tau \leq C_{0}^{(2)}, \quad \forall t>0 .
$$


By Eq. (1.2), Sobolev's imbedding theorem, and Young's inequality, we have

$$
\begin{aligned}
\left\|v_{x x}\right\| & \leq C_{0}^{(1)}\left(\left\|v_{t}\right\|+\|\theta\|_{H^{1}}+\left\|u_{x}\right\|+\left\|v_{x} u_{x}\right\|\right) \\
& \leq C_{0}^{(1)}\left(\left\|v_{t}\right\|+\left\|v_{x}\right\|^{1 / 2}\left\|v_{x x}\right\|^{1 / 2}\left\|u_{x}\right\|+1\right) \\
& \leq \frac{1}{2}\left\|v_{x x}\right\|+C_{0}^{(1)}\left(\left\|v_{t}\right\|+1\right)
\end{aligned}
$$

Combining (2.13) with (2.14) yields

$$
\left\|v_{x x}\right\| \leq C_{0}^{(2)}, \quad\left\|v_{x}\right\|_{L^{\infty}} \leq C_{0}^{(2)}, \quad \forall t>0 .
$$

By Eq. (1.3), in the same manner we have

$$
\begin{aligned}
\frac{d}{d t}\left\|\theta_{t}\right\|^{2} & +\frac{1}{C_{0}^{(1)}}\left\|\theta_{x t}\right\|^{2} \\
\leq & \frac{1}{2 C_{0}^{(1)}}\left\|\theta_{x t}\right\|^{2}+C_{0}^{(1)}\left(\left\|\theta_{x}\right\|^{2}\left\|v_{x}\right\|\left\|v_{x x}\right\|+\left\|v_{x}\right\|\left\|v_{x x}\right\|\left\|v_{x t}\right\|^{2}\right. \\
& \left.\quad+\left\|\theta_{t}\right\|^{2}+\left\|v_{x}\right\|^{2}+\left\|v_{x}\right\|^{1 / 2}\left\|v_{x x}\right\|^{1 / 2}\left\|\theta_{t}\right\|^{2}+\left\|v_{x t}\right\|^{2}+\left\|v_{x}\right\|^{3}\left\|v_{x x}\right\|\right) \\
\leq & \frac{1}{2 C_{0}^{(1)}}\left\|\theta_{x t}\right\|^{2}+C_{0}^{(2)}\left(\left\|\theta_{x}\right\|^{2}+\left\|v_{x t}\right\|^{2}+\left\|\theta_{t}\right\|^{2}+\left\|v_{x}\right\|^{2}\right) .
\end{aligned}
$$

Integrating (2.16) with respect to $t$, then applying (2.13) and Lemma 2.1, we get

$$
\left\|\theta_{t}\right\|^{2}+\int_{0}^{t}\left\|\theta_{x t}\right\|^{2}(\tau) d \tau \leq C_{0}^{(2)}, \quad \forall t>0 .
$$

Therefore, it follows from Eq. (1.3) that

$$
\left\|\theta_{x x}\right\| \leq C_{0}^{(2)}, \quad \forall t>0 .
$$

In what follows we are going to estimate the norm of $u$ in $H^{2}$. By Lemmas 2.1 and 2.2 , we easily get

$$
\begin{gathered}
\|B(x, t)\|_{L^{\infty}} \leq C_{0}^{(1)}, \quad|\exp (B(x, t))| \leq C_{0}^{(1)}, \\
\frac{1}{C_{0}^{(1)} \leq} D(x, t), \quad D^{-1}(x, t) \leq C_{0}^{(1)}, \quad \frac{1}{C_{0}^{(1)}} \leq Z(t) \leq 1, \\
\int_{0}^{t}\left(Z(t) Z^{-1}(\tau)\right)^{p} d \tau \leq C_{0}^{(1)}, \quad 0<p<+\infty .
\end{gathered}
$$

Hence, by Lemma 2.1,

$$
\begin{aligned}
\left\|D_{x}\right\| & \leq C_{0}^{(1)}, \\
\left\|D_{x x}\right\| & \leq C_{0}^{(2)}+C_{0}^{(1)}\left(\|v\|_{H^{1}}+\left\|v_{0}\right\|_{H^{1}}\right) \leq C_{0}^{(2)} .
\end{aligned}
$$

A straightforward calculation gives

$$
u_{x x}=I_{1}+I_{2}+I_{3}
$$


where

$$
\begin{aligned}
& I_{1}= D_{x x}(x, t) Z(t) \\
& \begin{aligned}
I_{2}=2 D_{x}(x, t) Z(t) & \int_{0}^{t} Z^{-1}(\tau)\left(\theta_{x}(x, \tau) D^{-1}(x, \tau)\right. \\
& \left.\quad-D_{x}(x, \tau) \theta(x, \tau) D^{-2}(x, \tau)\right) d \tau
\end{aligned} \\
& \begin{aligned}
I_{3}=D(x, t) Z(t) & \int_{0}^{t} Z^{-1}(\tau)\left(\theta_{x x}(x, \tau) D^{-1}(x, \tau)-2 \theta_{x}(x, \tau) D_{x}(x, \tau) D^{-2}(x, \tau)\right. \\
& \left.-\theta(x, \tau) D_{x x}(x, \tau) D^{-2}(x, \tau)+2 \theta(x, \tau) D_{x}^{2}(x, \tau) D^{-3}(x, \tau)\right) d \tau .
\end{aligned}
\end{aligned}
$$

Thus by Lemmas 2.1-2.2 and (2.15), (2.18), and (2.20)-(2.23), we get

$$
\begin{aligned}
\left\|I_{1}\right\|^{2} \leq & 2\left(\left\|D_{x x} Z\right\|^{2}+\int_{0}^{1}\left|D_{x x}(x, t)\right|^{2}\left(\int_{0}^{t} \theta(x, \tau) D^{-1}(x, \tau) Z(t) Z^{-1}(\tau) d \tau\right)^{2} d x\right) \\
\leq & C_{0}^{(2)} \\
\left\|I_{2}\right\|^{2} \leq & C_{0}^{(2)}\left\|D_{x}\right\|^{2}\left(\int_{0}^{t} Z(t) Z^{-1}(\tau) d \tau\right)^{2} \leq C_{0}^{(2)} \\
\left\|I_{3}\right\|^{2} \leq & C_{0}^{(1)} \int_{0}^{1} \int_{0}^{t} Z^{2}(t) Z^{-2}(\tau) d \tau \int_{0}^{t}\left(\theta_{x x}^{2}(x, \tau)+\theta_{x}^{2}(x, \tau)\right) d \tau d x \\
& +C_{0}^{(1)} \int_{0}^{1} \int_{0}^{t} Z(t) Z^{-1}(\tau) d \tau \int_{0}^{t} D_{x x}^{2}(x, \tau) Z(t) Z^{-1}(\tau) d \tau d x \\
& +C_{0}^{(1)} \int_{0}^{1}\left(\int_{0}^{t} Z(t) Z^{-1}(\tau) d \tau\right)^{2} d x \\
\leq & C_{0}^{(2)} .
\end{aligned}
$$

Combining (2.28)-(2.30) with (2.24) yields

$$
\left\|u_{x x}(t)\right\| \leq C_{0}^{(2)}, \quad \forall t>0 .
$$

Thus, (2.10) follows from (2.31), (2.18), (2.15), and Lemma 2.1.

The continuity of $S(t)$ with respect to $\left(u_{0}, v_{0}, \theta_{0}\right)$ in $H^{2}$ can be proved in the same manner as that for Lemma 2.3, hence can be omitted here. Thus, the proof of Theorem 2.1 is completed.

3. Existence of absorbing set in $H_{\beta}^{(1)}$. In this section we are going to prove the existence of an absorbing set in $H_{\beta}^{(1)}$. Throughout this section we always assume that the initial data belong to a bounded set of $H_{\beta}^{(1)}$. As mentioned in the introduction, first we have to prove that the orbit starting from any bounded set in $H_{\beta}^{(1)}$ will reenter $H_{\beta}^{(1)}$ after a finite time which should be uniform with respect to all orbits starting from that bounded set. 
LEMmA 3.1. If $\left(u_{0}, v_{0}, \theta_{0}\right) \in H_{\beta}^{(1)}$, then the following estimates hold:

$$
\begin{gathered}
\beta_{3} \leq \int_{0}^{1} u(x, t) d x=\int_{0}^{1} u_{0}(x) d x \leq \beta_{4}, \forall t>0, \\
\beta_{5} \leq \int_{0}^{1}\left(\theta+\frac{v^{2}}{2}\right)(x, t) d x=\int_{0}^{1}\left(\theta_{0}+\frac{v_{0}^{2}}{2}\right)(x) d x \leq \beta_{2}, \forall t>0, \\
-\int_{0}^{1}(\ln \theta+\ln u)(x, t) d x+\int_{0}^{t} \int_{0}^{1}\left(\frac{\theta_{x}^{2}}{u \theta^{2}}+\frac{v_{x}^{2}}{u \theta}\right)(x, \tau) d x d \tau \\
=-\int_{0}^{1}\left(\ln \theta_{0}+\ln u_{0}\right)(x) d x \leq-\beta_{1}, \quad \forall t>0 .
\end{gathered}
$$

Proof. See, e.g., [11], [16].

Lemma 3.2. If $\left(u_{0}, v_{0}, \theta_{0}\right) \in H_{\beta}^{(1)}$, then the following estimates hold:

$$
\begin{gathered}
\int_{0}^{t} \int_{0}^{1}\left(\frac{\theta_{x}^{2}}{u \theta^{2}}+\frac{v_{x}^{2}}{u \theta}\right)(x, \tau) d x d \tau \leq C_{\beta}, \quad \forall t>0, \\
\|v(t)\| \leq C_{\beta}, \quad \forall t>0, \\
0<C_{\beta}^{-1} \leq \int_{0}^{1} \theta^{\gamma}(x, t) d x \leq C_{\beta}, \quad \gamma \in[0,1], \quad \forall t>0 .
\end{gathered}
$$

Proof. See, e.g., [11], [16].

LEMma 3.3. If $\left(u_{0}, v_{0}, \theta_{0}\right) \in H_{\beta}^{(1)}$, then

$$
C_{\beta}^{-1} \leq u(x, t) \leq C_{\beta}, \quad \forall(x, t) \in[0,1] \times[0,+\infty) .
$$

Proof. We deduce from Lemma 2.2 and Lemmas 3.1-3.2 that

$$
\begin{gathered}
|B(x, t)| \leq C_{\beta}, \quad C_{\beta}^{-1} \leq D(x, t) \leq C_{\beta}, \quad \forall(x, t) \in[0,1] \times[0,+\infty), \\
C_{\beta}^{-1} \leq\left(\int_{0}^{1} u_{0}(x) d x\right)^{-1} \int_{0}^{1}\left(v^{2}+\theta\right)(x, \tau) d x \leq C_{\beta}, \quad \forall \tau \geq 0 \\
e^{-C_{\beta} t} \leq Z(t) \leq e^{-C_{\beta}^{-1} t}, \quad \forall t>0 \\
e^{-C_{\beta}(t-\tau)} \leq Z(t) Z^{-1}(\tau) \leq e^{-C_{\beta}^{-1}(t-\tau)}, \quad t \geq \tau \geq 0
\end{gathered}
$$

By Lemma 3.2, for any $t \geq 0$ there exists a point $a(t) \in[0,1]$ such that

$$
C_{\beta}^{-1} \leq \int_{0}^{1} \theta(x, t) d x=\theta(a(t), t) \leq C_{\beta} \text {. }
$$

Therefore,

$$
\left|\theta^{1 / 2}(x, t)-\theta^{1 / 2}(a(t), t)\right| \leq\left(\int_{0}^{1} \frac{\theta_{x}^{2}}{u \theta^{2}} d x\right)^{1 / 2}\left(\int_{0}^{1} u \theta d x\right)^{1 / 2} \leq C_{\beta}^{1 / 2} V^{1 / 2}(t) M_{u}^{1 / 2}(t)
$$

with

$$
V(t)=\int_{0}^{1} \frac{\theta_{x}^{2}}{u \theta^{2}} d x \quad \text { and } \quad M_{u}(t)=\max _{x \in[0,1]} u(x, t)
$$


Thus, for all $x \in[0,1]$,

$$
C_{\beta}-C_{\beta} M_{u}(t) V(t) \leq \theta(x, t) \leq C_{\beta}+C_{\beta} M_{u}(t) V(t) .
$$

By (2.6) and (3.8)-(3.14), we have

$$
u(x, t) \leq C_{\beta} e^{-C_{\beta}^{-1} t}+C_{\beta} \int_{0}^{t} \theta(x, \tau) e^{-C_{\beta}^{-1}(t-\tau)} d \tau .
$$

Therefore,

$$
M_{u}(t) \leq C_{\beta}+C_{\beta} \int_{0}^{t} M_{u}(\tau) V(\tau) d \tau
$$

By Gronwall's inequality and (3.4) we get

$$
M_{u}(t) \leq C_{\beta} \exp \left(C_{\beta} \int_{0}^{t} V(\tau) d \tau\right) \leq C_{\beta}
$$

On the other hand, by (2.6) and (3.8)-(3.11), we have

$$
u(x, t) \geq C_{\beta}^{-1} e^{-C_{\beta} t} .
$$

In what follows we will use a contradiction argument to prove that

$$
u(x, t) \geq C_{\beta}^{-1}, \quad \forall(x, t) \in[0,1] \times[0,+\infty) .
$$

In fact, if the above assertion is not true, then there exists a sequence of solutions $\left(u_{n}, v_{n}, \theta_{n}\right)$ with the initial data $\left(u_{n 0}, v_{n 0}, \theta_{n 0}\right) \in H_{\beta}^{(1)}$ converging weakly in $H^{1}$, strongly in $C[0,1]$ to $\left(u_{0}, v_{0}, \theta_{0}\right) \in H_{\beta}^{(1)}$ such that for the corresponding solution $(u, v, \theta)$ to $\left(u_{0}, v_{0}, \theta_{0}\right), \inf _{x \in[0,1], t \geq 0} u=0$. Thus there exists $\left(x_{n}, t_{n}\right) \in[0,1] \times[0,+\infty)$ such that as $n \rightarrow+\infty$,

$$
u\left(x_{n}, t_{n}\right) \rightarrow 0
$$

If the sequence $t_{n}$ has a subsequence, still denoted by $t_{n}$, converging to infinity, then by the result on the asymptotic behaviour in Lemma 2.2, as $n \rightarrow+\infty$,

$$
u\left(x_{n}, t_{n}\right) \rightarrow \int_{0}^{1} u_{0}(x) d x \geq \beta_{3}>0,
$$

which contradicts (3.20). If the sequence $t_{n}$ is bounded, i.e., there exists a constant $M>0$ independent of $n$ such that $0<t_{n} \leq M$, then by (3.18),

$$
u\left(x_{n}, t_{n}\right) \geq C_{\beta}^{-1} e^{-C_{\beta} t_{n}} \geq C_{\beta}^{-1} e^{-C_{\beta} M}>0,
$$

which again contradicts (3.20). Thus the proof is complete.

The next lemma is concerned with the boundedness of $\theta$ from below.

LEMMA 3.4. If $\left(u_{0}, v_{0}, \theta_{0}\right) \in H_{\beta}^{(1)}$, then

$$
C_{\beta}^{-1} \leq \theta(x, t), \quad \forall(x, t) \in[0,1] \times[0,+\infty) .
$$


Proof. Let $w=1 / \theta$. Then Eq. (1.3) can be rewritten as follows:

$$
w_{t}=\left(\rho w_{x}\right)_{x}-\left[2 \rho \theta w_{x}^{2}+\rho w^{2}\left(v_{x}-\theta / 2\right)^{2}\right]+\rho / 4
$$

with $\rho=1 / u$. Multiplying (3.24) by $2 r w^{2 r-1}$ with $r$ being an arbitrary natural number, and integrating the resultant over $\Omega=(0,1)$, by the fact that the expression in the square bracket is nonnegative and by Hölder's inequality, we get

$$
\begin{aligned}
\|w(t)\|_{L^{2 r}}^{2 r-1} \frac{d}{d t}\|w(t)\|_{L^{2 r}} & \leq \frac{1}{4} \int_{0}^{1} \rho w^{2 r-1} d x \\
& \leq C_{\beta}\|\rho\|_{L^{2 r}}\|w(t)\|_{L^{2 r}}^{2 r-1} \leq C_{\beta}\|w(t)\|_{L^{r-2}}^{2 r-1},
\end{aligned}
$$

which implies, by taking $r \rightarrow+\infty$, that

$$
\|w(t)\|_{L^{\infty}} \leq\left\|1 / \theta_{0}\right\|_{L^{\infty}}+C_{\beta} t \leq C_{\beta}(1+t) .
$$

Thus, for all $x \in[0,1], t \geq 0$,

$$
\theta(x, t) \geq \frac{1}{C_{\beta}(1+t)}
$$

Noting the asymptotic behaviour of $\theta$ in Lemma 2.1, Lemma 3.1, and (3.27), in the same manner as in the proof of (3.19), we can easily derive (3.23).

Lemma 3.5. For initial data belonging to a bounded set of $H_{\beta}^{(1)}$, there is $t_{0}>0$ depending only on boundedness of this bounded set such that for all $t \geq t_{0}, x \in[0,1]$,

$$
\frac{\beta_{5}}{2} \leq \theta(x, t) \leq 2 \beta_{2}, \quad \frac{\beta_{3}}{2} \leq u(x, t) \leq 2 \beta_{4} .
$$

Proof. It can be seen from (2.4) and (3.2) that as $t \rightarrow+\infty$,

$$
\int_{0}^{1} \theta(x, t) d x \rightarrow \int_{0}^{1}\left(\theta_{0}+\frac{v_{0}^{2}}{2}\right) d x, \quad\left\|\theta-\int_{0}^{1} \theta d x\right\|_{L^{\infty}} \rightarrow 0 .
$$

Now we use a contradiction argument to prove (3.28). Suppose that it is not true. Then there exists a sequence $t_{n} \rightarrow+\infty$ such that for all $x \in[0,1]$,

$$
\sup \theta\left(x, t_{n}\right)>2 \beta_{2}
$$

where sup is taken for all initial data in a given bounded set of $H_{\beta}^{(1)}$. Then in the same manner as for the proof of Lemma 3.3, there is $\left(u_{0}, v_{0}, \theta_{0}\right)$ belonging to this bounded set such that for the corresponding solution $(u, v, \theta)$, we have

$$
\theta\left(x, t_{n}\right) \geq 2 \beta_{2}, \quad \forall x \in[0,1] .
$$

This contradicts (3.29) and (3.2). In the same way, we can derive other parts of (3.28).

Thus the proof is completed.

It follows from Lemma 3.1 and Lemma 3.5 that for initial data belonging to a given bounded set of $H_{\beta}^{(1)}$, the orbit will reenter $H_{\beta}^{(1)}$ after a finite time. In what follows we are going to prove that there is an absorbing set in $H_{\beta}^{(1)}$. 
Let

$$
\begin{gathered}
E(u, v, S)=\frac{v^{2}}{2}+e(u, S)-e(\bar{u}, \bar{S})-\frac{\partial e}{\partial u}(\bar{u}, \bar{S})(u-\bar{u})-\frac{\partial e}{\partial S}(\bar{u}, \bar{S})(S-\bar{S}), \\
S=\log \theta+\log u \quad(\text { entropy), } \\
e(u, S)=\theta=\theta(u, S)=\frac{\exp (S)}{u} \quad \text { (internal energy), }
\end{gathered}
$$

where $\bar{S}, \bar{u}$, and $\bar{\theta}$ are constants and defined as follows:

$$
\begin{gathered}
\bar{S}=\log \bar{\theta}+\log \bar{u}, \quad \bar{u}=\int_{0}^{1} u(x, t) d x=\int_{0}^{1} u_{0}(x) d x \\
\beta_{5} \leq \bar{\theta}=\int_{0}^{1}\left(\theta+\frac{v^{2}}{2}\right)(x, t) d x=\int_{0}^{1}\left(\theta_{0}+\frac{v_{0}^{2}}{2}\right)(x) d x \leq \beta_{2} .
\end{gathered}
$$

Since we assume that the initial data $\left(u_{0}, v_{0}, \theta_{0}\right)$ belong to an arbitrarily bounded set of $H_{\beta}^{(1)}$, there is a positive constant $B$ such that $\left\|\left(u_{0}, v_{0}, \theta_{0}\right)\right\|_{H^{1}} \leq B$. We use $C_{\beta, B}$ to denote universal positive constants depending on $B$ and $\beta_{i}(i=1, \ldots, 5)$. Thus we have LEMma 3.6. The following inequalities hold:

$$
\frac{v^{2}}{2}+\frac{1}{C_{\beta}}\left(|u-\bar{u}|^{2}+|S-\bar{S}|^{2}\right) \leq E(u, v, S) \leq \frac{v^{2}}{2}+C_{\beta, B}\left(|u-\bar{u}|^{2}+|S-\bar{S}|^{2}\right) .
$$

Proof. By the mean value theorem and (3.32), there exists a point $(\tilde{u}, \tilde{S})$ between $(u, S)$ and $(\bar{u}, \bar{S})$ such that

$$
\begin{array}{r}
E(u, v, S)=\frac{v^{2}}{2}+\frac{1}{2}\left(\frac{\partial^{2} e}{\partial u^{2}}(\tilde{u}, \tilde{S})(u-\bar{u})^{2}+2 \frac{\partial^{2} e}{\partial u \partial S}(\tilde{u}, \tilde{S})(u-\bar{u})(S-\bar{S})\right. \\
\left.+\frac{\partial^{2} e}{\partial S^{2}}(\tilde{u}, \tilde{S})(S-\bar{S})^{2}\right) \\
=\frac{v^{2}}{2}+\frac{1}{2}\left(2 \tilde{u}^{-3} e^{\tilde{S}}(u-\bar{u})^{2}-2 \tilde{u}^{-2} e^{\tilde{S}}(u-\bar{u})(S-\bar{S})\right. \\
\left.+\tilde{u}^{-1} e^{\tilde{S}}(S-\bar{S})^{2}\right)
\end{array}
$$

where

$$
\begin{aligned}
& \tilde{u}=\lambda \bar{u}+(1-\lambda) u, \quad 0 \leq \lambda \leq 1, \\
& \tilde{S}=\lambda \bar{S}+(1-\lambda) S, \quad 0 \leq \lambda \leq 1 .
\end{aligned}
$$

It follows from Lemma 2.1 that $\|\theta\|_{L^{\infty}} \leq C_{\beta, B}$. Thus we deduce from Lemma 3.3 that

$$
\begin{gathered}
\frac{1}{C_{\beta}} \leq \tilde{u} \leq C_{\beta}, \quad\left|e^{\tilde{S}}\right| \leq C_{\beta, B}, \\
\left|\frac{\partial^{2} e}{\partial u^{2}}(\tilde{u}, \tilde{S})(u-\bar{u})^{2}\right| \leq C_{\beta, B}(u-\bar{u})^{2}, \\
\left|\frac{\partial^{2} e}{\partial u \partial S}(\tilde{u}, \tilde{S})(u-\bar{u})(S-\bar{S})\right| \leq C_{\beta, B}\left((u-\bar{u})^{2}+(S-\bar{S})^{2}\right), \\
\left|\frac{\partial^{2} e}{\partial S^{2}}(\tilde{u}, \tilde{S})(S-\bar{S})^{2}\right| \leq C_{\beta, B}(S-\bar{S})^{2},
\end{gathered}
$$


which together with (3.38) yield

$$
E(u, v, S) \leq \frac{v^{2}}{2}+C_{\beta, B}\left((u-\bar{u})^{2}+(S-\bar{S})^{2}\right) .
$$

On the other hand, we can deduce from Lemma 3.3 and Lemma 3.4 that

$$
\tilde{S} \geq C_{\beta}, \quad e^{\tilde{S}} \geq C_{\beta}
$$

Thus, it follows from Young's inequality that

$$
\left|\tilde{u}^{-2}(u-\bar{u})(S-\bar{S})\right| \leq \frac{3}{4} \tilde{u}^{-3}(u-\bar{u})^{2}+\frac{1}{3} \tilde{u}^{-1}(S-\bar{S})^{2},
$$

and it follows from (3.38) that

$$
\begin{aligned}
E(u, v, S) & \geq \frac{v^{2}}{2}+\frac{1}{4} \tilde{u}^{-3} e^{\tilde{S}}(u-\bar{u})^{2}+\frac{1}{6} \tilde{u}^{-1} e^{\tilde{S}}(S-\bar{S})^{2} \\
& \geq \frac{v^{2}}{2}+\frac{1}{C_{\beta}}\left((u-\bar{u})^{2}+(S-\bar{S})^{2}\right) .
\end{aligned}
$$

Thus the proof is completed.

Lemma 3.7. There exists a positive constant $\alpha_{1}=\alpha_{1}\left(C_{\beta, B}\right)>0$ such that for any fixed $\alpha \in\left(0, \alpha_{1}\right]$, the following estimates hold:

$$
\begin{aligned}
& e^{\alpha t}\left(\|v(t)\|^{2}+\|u(t)-\bar{u}\|^{2}+\|\theta(t)-\bar{\theta}\|^{2}+\left\|u_{x}\right\|^{2}\right) \\
& \quad+\int_{0}^{t} e^{\alpha \tau}\left(\left\|\theta^{\frac{1}{2}} u_{x}\right\|^{2}+\left\|\theta^{\frac{1}{2}} \rho_{x}\right\|^{2}+\left\|\theta_{x}\right\|^{2}+\left\|v_{x}\right\|^{2}\right)(\tau) d \tau \leq C_{\beta, B}, \quad \forall t>0 .
\end{aligned}
$$

Proof. By $\rho=1 / u$ and Eqs. (1.1)-(1.3), it is easy to verify that $(\rho, v, S)$ satisfies

$$
\begin{aligned}
& \left(\theta+\frac{v^{2}}{2}\right)_{t}+\left(\rho \theta v-\rho \theta_{x}-\rho v v_{x}\right)_{x}=0, \\
& S_{t}-\left(\rho \theta_{x} / \theta\right)_{x}-\rho\left(\theta_{x} / \theta\right)^{2}-\rho v_{x}^{2} / \theta=0 .
\end{aligned}
$$

Since $\bar{u}_{t}=0, \bar{\theta}_{t}=0$, we have

$$
\begin{gathered}
E_{t}\left(\rho^{-1}, v, S\right)+\frac{\rho \bar{\theta}}{\theta}\left(v_{x}^{2}+\frac{\theta_{x}^{2}}{\theta}\right)=\left[\rho v v_{x}+(1-\bar{\theta} / \theta) \rho \theta_{x}-(\rho \theta-\bar{\rho} \bar{\theta}) v\right]_{x} \\
{\left[\left(\rho_{x} / \rho\right)^{2} / 2+\rho_{x} v / \rho\right]_{t}+\theta \rho_{x}^{2} / \rho=\rho v_{x}^{2}-\rho_{x} \theta_{x}-\left(\rho v v_{x}\right)_{x}}
\end{gathered}
$$

with $\bar{\rho}=1 / \bar{u}$.

Multiplying (3.52), (3.53) by $e^{\alpha t}, \eta e^{\alpha t}$, respectively, and then adding the resultants, we get

$$
\begin{aligned}
& \frac{\partial}{\partial t}\left(e ^ { \alpha t } \left(E+\eta\left(\rho_{x} / \rho\right)^{2} / 2\right.\right.\left.\left.+\eta \rho_{x} v / \rho\right)\right) \\
&+e^{\alpha t} \bar{\theta} \rho\left(v_{x}^{2}+\theta_{x}^{2} / \theta\right) / \theta \\
&+\eta e^{\alpha t}\left(\theta \rho_{x}^{2} / \rho+\rho_{x} \theta_{x}-\rho v_{x}^{2}\right) \\
&=\alpha e^{\alpha t}\left(E+\eta\left(\rho_{x} / \rho\right)^{2} / 2+\eta \rho_{x} v / \rho\right) \\
&+e^{\alpha t}\left((1-\eta) \rho v v_{x}+(1-\bar{\theta} / \theta) \rho \theta_{x}-(\rho \theta-\bar{\rho} \bar{\theta}) v\right)_{x} .
\end{aligned}
$$


Integrating (3.54) over $Q_{t}=[0,1] \times[0, t]$, by Lemmas 3.3, 3.4, and 3.6, Young's inequality and the boundary conditions (1.4), we obtain that

$$
\begin{aligned}
L \equiv & e^{\alpha t} \int_{0}^{1}\left(E+\eta\left(\rho_{x} / \rho\right)^{2} / 2+\eta \rho_{x} v / \rho\right) d x+\int_{0}^{t} \int_{0}^{1} e^{\alpha \tau} \bar{\theta} \rho\left(v_{x}^{2}+\theta_{x}^{2} / \theta\right) / \theta(x, \tau) d x d \tau \\
& +\eta \int_{0}^{t} \int_{0}^{1} e^{\alpha \tau}\left(\theta \rho_{x}^{2} / \rho+\rho_{x} \theta_{x}-\rho v_{x}^{2}(x, \tau) d x d \tau\right. \\
\leq & C_{\beta, B}+\alpha \int_{0}^{t} \int_{0}^{1} e^{\alpha \tau}\left(\frac{v^{2}}{2}+C_{\beta, B}\left(|u-\bar{u}|^{2}+|S-\bar{S}|^{2}\right)+\eta\left(\rho_{x} / \rho\right)^{2} / 2+\eta \rho_{x} v / \rho\right) d x d \tau \\
\leq & C_{\beta, B}+\alpha \int_{0}^{t} \int_{0}^{1} e^{\alpha \tau}\left((1+\eta) \frac{v^{2}}{2}+C_{\beta, B}\left(|u-\bar{u}|^{2}+|S-\bar{S}|^{2}\right)+\eta\left(\rho_{x} / \rho\right)^{2}\right) d x d \tau .
\end{aligned}
$$

On the other hand, by Young's inequality and Lemma 3.6, we can easily get

$$
\begin{aligned}
L \geq & e^{\alpha t} \int_{0}^{1}\left(\left(\frac{1}{2}-\eta\right) v^{2}+\frac{1}{C_{\beta}}\left((u-\bar{u})^{2}+(S-\bar{S})^{2}+\eta\left(\rho_{x} / \rho\right)^{2} / 4\right)\right) d x \\
& +\int_{0}^{t} \int_{0}^{1} e^{\alpha \tau}\left(\left(\frac{\bar{\theta}}{\theta}-\eta\right) \rho v_{x}^{2}+\left(\frac{\bar{\theta}}{\theta}-\frac{\eta}{2}\right) \rho \theta_{x}^{2} / \theta+\eta \theta \rho_{x}^{2} /(2 \rho)\right) d x d \tau \\
\leq & \frac{1}{C_{\beta}} e^{\alpha t} \int_{0}^{1}\left(v^{2}+(u-\bar{u})^{2}+(S-\bar{S})^{2}+\eta \rho_{x}^{2}\right) d x \\
& +\frac{1}{C_{\beta, B}} \int_{0}^{t} \int_{0}^{1} e^{\alpha \tau}\left(v_{x}^{2}+\theta_{x}^{2}+\theta \rho_{x}^{2}+\theta u_{x}^{2}\right) d x d \tau,
\end{aligned}
$$

where we take $\eta$ so small that $0<\eta<\frac{1}{2}$, and $\bar{\theta} / \theta-\eta \geq \beta_{5} / C_{B, B}-\eta>0$.

By the mean value theorem, we have

$$
S-\bar{S}=\frac{1}{\theta_{1}}(\theta-\bar{\theta})+\frac{1}{u_{1}}(u-\bar{u}),
$$

where $\frac{1}{C_{\beta}} \leq \min (u, \bar{u}) \leq u_{1} \leq \max (u, \bar{u}) \leq C_{\beta}, \frac{1}{C_{\beta}} \leq \min (\theta, \bar{\theta}) \leq \theta_{1} \leq \max (\theta, \bar{\theta}) \leq C_{\beta, B}$.

By Poincaré's inequality we get

$$
\begin{aligned}
\|S-\bar{S}\| & \leq C_{\beta}(\|u-\bar{u}\|+\|\theta-\bar{\theta}\|) \\
& \leq C_{\beta}\left(\left\|u_{x}\right\|+\left\|\theta_{x}\right\|\right),
\end{aligned}
$$

and

$$
\|\theta-\bar{\theta}\| \leq C_{\beta, B}(\|S-\bar{S}\|+\|u-\bar{u}\|) .
$$

Thus, by (3.58)-(3.59) and Lemma 3.3, we get

$$
\begin{gathered}
e^{\alpha t}\left(\|v\|^{2}+\|u-\bar{u}\|^{2}+\|S-\bar{S}\|^{2}+\left\|\rho_{x}\right\|^{2}+\left\|u_{x}\right\|^{2}\right) \\
+\int_{0}^{t} e^{\alpha \tau}\left(\left\|v_{x}\right\|^{2}+\left\|\theta_{x}\right\|^{2}+\left\|\theta^{1 / 2} \rho_{x}\right\|^{2}+\left\|\theta^{1 / 2} u_{x}\right\|^{2}\right) d \tau \\
\quad \leq C_{\beta, B}+C_{\beta, B}^{\prime} \alpha \int_{0}^{t} e^{\alpha \tau}\left(\left\|v_{x}\right\|^{2}+\left\|\theta_{x}\right\|^{2}+\left\|u_{x}\right\|^{2}\right) d \tau
\end{gathered}
$$


By Lemma 3.4, we deduce that

$$
\begin{aligned}
& \left\|u_{x}\right\|^{2} \leq C_{\beta}\left\|\theta^{1 / 2} u_{x}\right\|^{2}, \\
& \left\|\rho_{x}\right\|^{2} \leq C_{\beta}\left\|\theta^{1 / 2} \rho_{x}\right\|^{2} .
\end{aligned}
$$

Thus, inserting (3.61) and (3.62) into (3.60) yields

$$
\begin{aligned}
& e^{\alpha t}\left(\|v\|^{2}+\|u-\bar{u}\|^{2}+\|S-\bar{S}\|^{2}+\left\|\rho_{x}\right\|^{2}+\left\|u_{x}\right\|^{2}\right) \\
& +\int_{0}^{t} e^{\alpha \tau}\left(\left\|v_{x}\right\|^{2}+\left\|\theta_{x}\right\|^{2}+\left\|\theta^{\frac{1}{2}} \rho_{x}\right\|^{2}+\left\|\theta^{\frac{1}{2}} u_{x}\right\|^{2}\right) d \tau \\
& \quad \leq C_{\beta, B}+C_{\beta, B}^{\prime} \alpha \int_{0}^{t} e^{\alpha \tau}\left(\left\|v_{x}\right\|^{2}+\left\|\theta_{x}\right\|^{2}+\left\|\theta^{\frac{1}{2}} u_{x}\right\|^{2}\right) d \tau,
\end{aligned}
$$

which implies that there exists a positive constant $\alpha_{1}=\alpha_{1}\left(C_{\beta, B}\right) \leq \frac{1}{2 C_{\beta, B}^{\prime}}$ such that when $\alpha \in\left(0, \alpha_{1}\right]$, it follows that

$$
\begin{aligned}
e^{\alpha t}\left(\|v\|^{2}+\|u-\bar{u}\|^{2}\right. & \left.+\|S-\bar{S}\|^{2}+\left\|\rho_{x}\right\|^{2}+\left\|u_{x}\right\|^{2}\right) \\
& +\int_{0}^{t} e^{\alpha \tau}\left(\left\|v_{x}\right\|^{2}+\left\|\theta_{x}\right\|^{2}+\left\|\theta^{\frac{1}{2}} \rho_{x}\right\|^{2}+\left\|\theta^{\frac{1}{2}} u_{x}\right\|^{2}\right) d \tau \leq C_{\beta, B} .
\end{aligned}
$$

Thus, combining it with (3.59) yields the desired result.

Lemma 3.8. There exists a positive constant $\alpha_{2}=\alpha_{2}\left(C_{\beta, B}\right) \leq \alpha_{1}$ such that for fixed $\alpha \in\left(0, \alpha_{2}\right]$, it follows that

$$
e^{\alpha t}\left(\left\|v_{x}(t)\right\|^{2}+\left\|\theta_{x}(t)\right\|^{2}\right)+\int_{0}^{t} e^{\alpha \tau}\left(\left\|v_{x x}\right\|^{2}+\left\|\theta_{x x}\right\|^{2}\right)(\tau) d \tau \leq C_{\beta, B}, \quad \forall t>0
$$

which together with the previous lemma implies that when $\alpha \in\left(0, \alpha_{2}\right]$, it follows that

$$
\|(u-\bar{u}, v, \theta-\bar{\theta})\|_{H^{1}} \leq C_{\beta, B} e^{-\alpha t}, \quad \forall t>0 .
$$

Proof. System (1.1) (1.3) can be rewritten as follows:

$$
\begin{aligned}
\rho_{t}+\rho^{2} v_{x} & =0, \\
v_{t}-\left(\rho v_{x}\right)_{x}-(\rho \theta)_{x} & =0, \\
\theta_{t}-\left(\rho \theta_{x}\right)_{x}+\rho \theta v_{x}-\rho v_{x}^{2} & =0 .
\end{aligned}
$$

Multiplying (3.68), (3.69) by $-e^{\alpha t} v_{x x},-e^{\alpha t} \theta_{x x}$, respectively, then integrating them over $Q_{t}$, and adding the resultants, by Young's inequality, the imbedding theorem and Lemma 
3.3 , we get

$$
\begin{aligned}
& \frac{1}{2} e^{\alpha t}\left(\left\|v_{x}\right\|^{2}+\left\|\theta_{x}\right\|^{2}\right)+\int_{0}^{t} \int_{0}^{1} e^{\alpha \tau} \rho\left(v_{x x}^{2}+\theta_{x x}^{2}\right)(x, \tau) d x d \tau \\
& \leq C_{\beta, B}+\frac{\alpha}{2} \int_{0}^{t}\left(\left\|v_{x}\right\|^{2}+\left\|\theta_{x}\right\|^{2}\right) e^{\alpha \tau} d \tau \\
& \quad-\int_{0}^{t} \int_{0}^{1} e^{\alpha \tau}\left(v_{x x}(\rho \theta)_{x}-\rho \theta v_{x} \theta_{x x}+\rho v_{x}^{2} \theta_{x x}+\rho_{x} v_{x} v_{x x}+\rho_{x} \theta_{x} \theta_{x x}\right) d x d \tau \\
& \leq C_{\beta, B}+\frac{\alpha}{2} \int_{0}^{t}\left(\left\|v_{x}\right\|^{2}+\left\|\theta_{x}\right\|^{2}\right) e^{\alpha \tau} d \tau+\frac{3}{4} \int_{0}^{t} \int_{0}^{1} e^{\alpha \tau} \rho\left(v_{x x}^{2}+\theta_{x x}^{2}\right) d x d \tau \\
&+\int_{0}^{t} \int_{0}^{1} e^{\alpha \tau} \rho^{-1}\left(\theta^{2} \rho_{x}^{2}+\rho^{2} \theta_{x}^{2}+\rho^{2} \theta^{2} v_{x}^{2}+\rho^{2} v_{x}^{4}+\rho_{x}^{2} v_{x}^{2}+\rho_{x}^{2} \theta_{x}^{2}\right) d x d \tau \\
& \leq C_{\beta, B}+\frac{\alpha}{2} \int_{0}^{t}\left(\left\|v_{x x}\right\|^{2}+\left\|\theta_{x x}\right\|^{2}\right) e^{\alpha \tau} d \tau+\frac{3}{4} \int_{0}^{t} \int_{0}^{1} e^{\alpha \tau} \rho\left(v_{x x}^{2}+\theta_{x x}^{2}\right) d x d \tau \\
&+C_{\beta, B} \int_{0}^{t} e^{\alpha \tau}\left(\left\|\theta^{\frac{1}{2}} \rho_{x}\right\|^{2}+\left\|\theta_{x}\right\|^{2}+\left\|v_{x}\right\|^{2}+\left\|v_{x}\right\|^{3}\left\|v_{x x}\right\|+\left\|v_{x}\right\|\left\|v_{x x}\right\|\left\|\rho_{x}\right\|^{2}\right. \\
& \leq C_{\beta, B}+\alpha \int_{0}^{t}\left(\left\|v_{x x}\right\|^{2}+\left\|\theta_{x x}\right\|^{2}\right) e^{\alpha \tau} d \tau+\frac{3}{4} \int_{0}^{t} \int_{0}^{1} e^{\alpha \tau} \rho\left(v_{x x}^{2}+\theta_{x x}^{2}\right) d x d \tau \\
&+C_{\beta} \varepsilon \int_{0}^{t} \int_{0}^{1} e^{\alpha \tau} \rho\left(v_{x x}^{2}+\theta_{x x}^{2}\right) d x d \tau \\
& \frac{C_{\beta, B}}{\varepsilon} \int_{0}^{t} e^{\alpha \tau}\left(\left\|\theta^{\frac{1}{2}} \rho_{x}\right\|^{2}+\left\|\theta_{x}\right\|^{2}+\left\|v_{x}\right\|^{2}\right) d \tau .
\end{aligned}
$$

Choose $\varepsilon$ small enough. Then it follows from (3.70) and Lemmas 3.3 and 3.7 that

$$
\begin{array}{r}
e^{\alpha t}\left(\left\|v_{x}\right\|^{2}+\left\|\theta_{x}\right\|^{2}\right)+\int_{0}^{t} e^{\alpha \tau}\left(\left\|v_{x x}\right\|^{2}+\left\|\theta_{x x}\right\|^{2}\right) d \tau \\
\leq C_{\beta, B}+C_{\beta, B}^{\prime \prime} \alpha \int_{0}^{t}\left(\left\|v_{x x}\right\|^{2}+\left\|\theta_{x x}\right\|^{2}\right) e^{\alpha \tau} d \tau .
\end{array}
$$

Choosing $\alpha$ in (3.71) small enough such that $0<\alpha \leq \min \left(\alpha_{1}, C_{\beta, B}^{\prime \prime}\right)$, we get

$$
e^{\alpha t}\left(\left\|v_{x}\right\|^{2}+\left\|\theta_{x}\right\|^{2}\right)+\int_{0}^{t} e^{\alpha \tau}\left(\left\|v_{x x}\right\|^{2}+\left\|\theta_{x x}\right\|^{2}\right) d \tau \leq C_{\beta, B},
$$

which, together with Lemma 3.8, yields (3.65). Thus the proof is completed.

Finally, we have the following result on the existence of an absorbing ball in $H_{\beta}^{(1)}$.

TheOREM 3.1. Let $R_{1}=R_{1}(\beta)=4\left(\beta_{2}^{2}+\beta_{4}^{2}\right)$ and $B_{1}=\left\{(u, v, \theta) \in H_{\beta}^{(1)},\|(u, v, \theta)\|_{H^{(1)}} \leq\right.$ $\left.R_{1}\right\}$. Then $B_{1}$ is an absorbing ball in $H_{\beta}^{(1)}$.

Proof. From Lemma 3.1 and Lemma 3.8 we deduce that for any initial data belonging to a bounded set with $\left\|\left(u_{0}, v_{0}, \theta_{0}\right)\right\|_{H^{(1)}} \leq B$, there is $t_{1}$ depending on $B$ and $\beta$ such that 
when $t \geq t_{1}$,

$$
\begin{aligned}
\|(u, v, \theta)(t)\|_{H^{(1)}}^{2} & =\|u(t)\|_{H^{1}}^{2}+\|v(t)\|_{H^{1}}^{2}+\|\theta(t)\|_{H^{1}}^{2} \\
& \leq 2\left(\bar{u}^{2}+\bar{\theta}^{2}+\|u-\bar{u}\|_{H^{1}}^{2}+\|v\|_{H^{1}}^{2}+\|\theta-\bar{\theta}\|_{H^{1}}^{2}\right) \\
& \leq 2\left(\beta_{2}^{2}+\beta_{4}^{2}\right)+C_{\beta, B}(\beta) e^{-\alpha t} \leq R_{0}^{2} .
\end{aligned}
$$

Thus, the proof is completed.

4. Existence of absorbing set in $H_{\beta}^{(2)}$. In this section we are going to prove existence of an absorbing set in $H_{\beta}^{(2)}$. Throughout this section we always assume that the initial data belong to a bounded set in $H_{\beta}^{(2)}$, i.e., $\left\|\left(u_{0}, v_{0}, \theta_{0}\right)\right\|_{H}^{(2)} \leq B$ with $B$ being any given positive constant. We first obtain the uniform estimates on $H^{2}$ norms of $v$ and $\theta$.

Lemma 4.1. There exists a positive constant $\alpha_{0}=\alpha\left(C_{\beta, B}\right) \leq \alpha_{2}\left(C_{\beta, B}\right)$ such that for any fixed $\alpha \in\left(0, \alpha_{0}\right]$, and for all $t>0$,

$$
\|\theta\|_{H^{2}}^{2}+\|v\|_{H^{2}}^{2} \leq \beta_{2}^{2}+C_{\beta, B} e^{-\alpha t} .
$$

REMARK 4.1. Let $t_{2}=t_{2}\left(C_{\beta, B}\right) \geq \max \left(t_{1}\left(C_{\beta, B}\right),-\alpha^{-1} \ln \left(\beta_{2}^{2} / C_{\beta, B}\right)\right)$. Then estimate (4.1) implies that for any $t \geq t_{2}\left(C_{\beta, B}\right)$,

$$
\|\theta(t)\|_{H^{2}}^{2}+\|v(t)\|_{H^{2}}^{2} \leq 2 \beta_{2}^{2}
$$

Proof of Lemma 4.1. Differentiating Eq. (1.2) with respect to $t$, then multiplying the resulting equation by $v_{t} e^{\alpha t}$ and integrating the resultant over $Q_{t}$, by Lemma 3.3 we get

$$
\begin{aligned}
& \frac{1}{2} e^{\alpha t}\left\|v_{t}(t)\right\|^{2}+\frac{1}{\tilde{C}_{\beta}} \int_{0}^{t} e^{\alpha \tau}\left\|v_{x t}\right\|^{2} d \tau \\
& \leq C_{\beta, B}+\frac{\alpha}{2} \int_{0}^{t} e^{\alpha \tau}\left\|v_{t}\right\|^{2} d \tau+C_{\beta} \int_{0}^{t} \int_{0}^{1}\left(v_{x}^{2}+\left|\theta_{t}\right|\right)\left|v_{x t}\right| e^{\alpha \tau} d x d \tau \\
& \leq C_{\beta, B}+\alpha \int_{0}^{t} e^{\alpha \tau}\left\|v_{x t}\right\|^{2} d \tau+\frac{C_{\beta}}{\alpha} \int_{0}^{t}\left\|\theta_{t}\right\|^{2} e^{\alpha \tau} d \tau
\end{aligned}
$$

It follows from Eq. (1.3) that

$$
\left\|\theta_{t}\right\| \leq C_{\beta, B}\left(\left\|\theta_{x x}\right\|+\left\|\theta_{x}\right\|+\left\|v_{x}\right\|+\left\|v_{x x}\right\|\right) .
$$

Let $\alpha_{0}=\min \left(\alpha_{2}\left(C_{\beta, B}\right), 1 /\left(2 \tilde{C}_{\beta}\right)\right)$ and let $\alpha$ be a positive constant such that $\alpha \in\left(0, \alpha_{0}\right]$. Then we deduce from (4.3)-(4.4) and Lemma 2.1 that

$$
\begin{aligned}
e^{\alpha t}\left\|v_{t}(t)\right\|^{2}+\int_{0}^{t} e^{\alpha \tau}\left\|v_{x t}\right\|^{2} d \tau & \leq C_{\beta, B}+C_{\beta, B, \alpha} \int_{0}^{t}\left\|\theta_{t}\right\|^{2} e^{\alpha \tau} d \tau \\
& \leq C_{\beta, B}
\end{aligned}
$$

By Eq. (1.2), we easily get

$$
\begin{aligned}
\left\|v_{x x}\right\| & \leq C_{\beta, B}\left(\left\|u_{x}\right\|+\left\|\theta_{x}\right\|+\left\|v_{x}\right\|+\left\|v_{t}\right\|\right) \\
& \leq C_{\beta, B}\left(\left\|u_{x}\right\|+\left\|\theta_{x}\right\|+\left\|v_{x}\right\|+\left\|v_{x t}\right\|\right),
\end{aligned}
$$


which, together with (4.5) and Lemmas (3.7)-(3.8), yields

$$
e^{\alpha t}\left(\left\|v_{t}(t)\right\|^{2}+\left\|v_{x x}(t)\right\|^{2}\right)+\int_{0}^{t} e^{\alpha \tau}\left\|v_{x t}\right\|^{2} d \tau \leq C_{\beta, B} .
$$

In the same manner, we can get

$$
e^{\alpha t}\left(\left\|\theta_{t}(t)\right\|^{2}+\left\|\theta_{x x}(t)\right\|^{2}\right)+\int_{0}^{t} e^{\alpha \tau}\left\|\theta_{x t}\right\|^{2} d \tau \leq C_{\beta, B}
$$

Thus, by Lemma (3.8) and (4.7)-(4.8), for any fixed $\alpha \in\left(0, \alpha_{0}\right]$, we have

$$
\|\theta-\bar{\theta}\|_{H^{2}}^{2}+\|v\|_{H^{2}}^{2} \leq C_{\beta, B} e^{-\alpha t}, \quad \forall t>0 .
$$

It turns out that

$$
\begin{aligned}
\|\theta\|_{H^{2}}^{2}+\|v\|_{H^{2}}^{2} & \leq \bar{\theta}^{2}+C_{\beta, B} e^{-\alpha t} \\
& \leq \beta_{2}^{2}+C_{\beta, B} e^{-\alpha t}, \quad \forall t>0 .
\end{aligned}
$$

Thus, the proof is completed.

Lemma 4.2. There exists a constant $t_{3}$ depending only on $\beta, B$ with $t_{3} \geq t_{2}$ such that when $t \geq t_{3}$, the following estimate holds:

$$
\begin{aligned}
& \|u(t)\|_{H^{1}}^{2}+\|v(t)\|_{H^{2}}^{2}+\|\theta(t)\|_{H^{2}}^{2}+\|v(t)\|_{L^{\infty}}+\|\theta(t)\|_{L^{\infty}} \\
& \quad+\int_{t_{3}}^{t}\left(\left\|u_{x}\right\|^{2}+\|v\|_{H^{2}}^{2}+\left\|\theta_{x}\right\|^{2}+\left\|\theta_{x x}\right\|^{2}+\left\|v_{x}\right\|_{L^{\infty}}^{2}+\left\|v_{x t}\right\|^{2}+\left\|\theta_{x t}\right\|^{2}\right)(\tau) d \tau \\
& \quad \leq 3\left(\beta_{2}^{2}+2 \beta_{4}^{2}\right) .
\end{aligned}
$$

Proof. The assertion easily follows from Lemma 3.8 and (4.1), (4.5), (4.7)-(4.8).

In what follows we get the estimate on $\left\|u_{x x}\right\|$.

LEMmA 4.3. For any given constant $\delta>0$, there exists a constant $t_{4}$ depending only on $\beta, B$ and $\delta$ with $t_{4} \geq t_{3}$ such that when $t \geq t_{4}$, the following estimate holds:

$$
\left\|u_{x x}\right\| \leq \delta
$$

Proof. Differentiating Eq. (1.2) with respect to $x$, then multiplying the resulting equation by $u_{x x}$, and integrating with respect to $x$, using Eq. (1.1), i.e., $u_{x x t}=v_{x x x}$, we get

$$
\begin{aligned}
& \frac{1}{2} \frac{d}{d t}\left\|u_{x x}\right\|^{2}+\int_{0}^{1} \frac{\theta}{u} u_{x x}^{2} d x \\
& \quad=\int_{0}^{1}\left(v_{x t} u+\theta_{x x}-\frac{2 \theta_{x} u_{x}}{u}+\frac{2 \theta u_{x}}{u^{2}}+\frac{2 v_{x x} u_{x}}{u}+\frac{v_{x} u_{x x}}{u^{2}}-\frac{2 v_{x} u_{x}^{2}}{u^{2}}\right) u_{x x} d x
\end{aligned}
$$

Let $\tilde{C}_{\beta}=\beta_{5} /\left(4 \beta_{4}\right)$. Then it follows from Lemma 3.5 that when $t \geq t_{2} \geq t_{0}$,

$$
\int_{0}^{1} \frac{\theta}{u} u_{x x}^{2} d x \geq \tilde{C}_{\beta}\left\|u_{x x}\right\|^{2}
$$


On the other hand, by Young's inequality, Lemma 3.8 and Lemma 4.1 , there is $t_{5} \geq t_{2}$ such that when $t \geq t_{5}$, we have

$$
\begin{aligned}
\left|\int_{0}^{1} v_{x t} u u_{x x} d x\right| & \leq \varepsilon\left\|u_{x x}\right\|^{2}+C_{\varepsilon, \beta}\left\|v_{x t}\right\|^{2}, \\
\left|\int_{0}^{1} u_{x x} \theta_{x x} d x\right| & \leq \frac{\varepsilon}{2}+\frac{1}{2 \varepsilon}\left\|\theta_{x x}\right\|^{2}, \\
\left|\int_{0}^{1} \frac{2 \theta_{x} u_{x}}{u} u_{x x} d x\right| & \leq \frac{\varepsilon}{2}\left\|u_{x x}\right\|^{2}+C_{\varepsilon, \beta}\left\|\theta_{x}\right\|^{2}, \\
\left|\int_{0}^{1} \frac{2 \theta u_{x}}{u^{2}} u_{x x} d x\right| & \leq \frac{\varepsilon}{2}\left\|u_{x x}\right\|^{2}+C_{\varepsilon, \beta}\left\|u_{x}\right\|^{2}, \\
\left|\int_{0}^{1} \frac{v_{x x} u_{x}}{u} u_{x x} d x\right| & \leq \frac{\varepsilon}{2}\left\|u_{x x}\right\|^{2}+C_{\varepsilon, \beta}\left\|v_{x x}\right\|^{2}, \\
\left|\int_{0}^{1} \frac{v_{x}}{u^{2}} u_{x x}^{2} d x\right| & \leq C_{\beta, B} e^{-\alpha t}\left\|u_{x x}\right\|^{2} \leq \frac{\tilde{C}_{\beta}}{4}\left\|u_{x x}\right\|^{2}, \\
\left|\int_{0}^{1} \frac{2 v_{x} u_{x}^{2}}{u^{2}} u_{x x} d x\right| & \leq \frac{\varepsilon}{2}\left\|u_{x x}\right\|^{2}+C_{\varepsilon, \beta}\left\|v_{x}\right\|^{2} .
\end{aligned}
$$

Choosing $\varepsilon$ small enough, we infer from (4.13)-(4.21) that when $t \geq t_{5}$,

$$
\frac{1}{2} \frac{d}{d t}\left\|u_{x x}\right\|^{2}+\frac{\tilde{C}_{\beta}}{2}\left\|u_{x x}\right\|^{2} \leq C_{\beta}\left(\left\|v_{x t}\right\|^{2}+\left\|\theta_{x x}\right\|^{2}+\left\|u_{x}\right\|^{2}+\left\|v_{x x}\right\|^{2}+\left\|v_{x}\right\|^{2}\right) .
$$

Now $\alpha$ is chosen such that $\alpha \leq \tilde{C}_{\beta} / 2$. It follows from (4.22), Lemma 2.3 and (4.6)-(4.8) that when $t \geq t_{5}$,

$$
\left\|u_{x x}(t)\right\|^{2} \leq\left\|u_{x x}\left(t_{5}\right)\right\|^{2} e^{-\tilde{C}_{\beta}\left(t-t_{5}\right)}+C_{\beta, B} e^{-\alpha t} \leq C_{\beta, B} e^{-\tilde{C}_{\beta}\left(t-t_{5}\right)}+C_{\beta, B} e^{-\alpha t} .
$$

Thus, it follows from (4.23) that there exists $t_{4} \geq t_{5}$ such that when $t \geq t_{4},(4.12)$ holds.

Let $R_{2}=2\left(\beta_{2}+2 \beta_{4}\right)$. Then we immediately infer from Lemmas 4.2 and 4.3 the following assertion.

TheOREM 4.1. The ball $B_{2}=\left\{(u, v, \theta) \in H_{\beta}^{(2)},\|(u, v, \theta)\|_{H^{2}}^{2} \leq R_{2}^{2}\right\}$ is an absorbing ball in $H_{\beta}^{(2)}$.

5. Proof of Theorem 1.1. Having proved the existence of absorbing balls in $H_{\beta}^{(2)}$ and $H_{\beta}^{(1)}$, we can exactly follow the abstract framework established in [2] by Ghidaglia to conclude that

LEMMA 5.1. The set

$$
\omega\left(B_{2}\right)=\bigcap_{s \geq 0} \overline{\bigcup_{t \geq s} S(t) B_{2}},
$$

where the closures are taken with respect to the weak topology of $H^{(2)}$, is included in $B_{2}$ and nonempty. It is invariant by $S(t)$, i.e.,

$$
S(t) \omega\left(B_{2}\right)=\omega\left(B_{2}\right), \quad \forall t>0 .
$$


REMARK 5.1. If we take $B$ to be a bounded set in $H_{\beta}^{(2)}$, we can also define $\omega(B)$ by (5.1) and when $B$ is nonempty, $\omega(B)$ is also included in $B_{2}$, nonempty and invariant. Since $B_{2}$ is an absorbing ball, it is clear that $\omega(B) \subseteq \omega\left(B_{2}\right)$. This shows that $\omega\left(B_{2}\right)$ is maximal in the sense of inclusion.

THEOREM 5.1. The set

$$
\mathcal{A}_{2, \beta}=\omega\left(B_{2}\right)
$$

satisfies

$$
\mathcal{A}_{2, \beta} \text { is bounded and weakly closed in } H_{\beta}^{(2)} \text {, }
$$

$$
S(t) \mathcal{A}_{2, \beta}=\mathcal{A}_{2, \beta}, \quad \forall t \geq 0,
$$

and, for every bounded set $B$ in $H_{\beta}^{(2)}$,

$$
\lim _{t \rightarrow+\infty} d^{w}\left(S(t) B, \mathcal{A}_{2, \beta}\right)=0 .
$$

Moreover, it is the maximal set in the sense of inclusion that satisfies (5.4), (5.5), and (5.6).

Proof. The proofs of Lemma 5.1 and Theorem 5.1 exactly follow the same argument as described in [2], pp. 387-389, using the facts that $S(t)$ is continuous in $H_{\beta}^{(2)}, H_{\beta}^{(1)}$, respectively, $H_{\beta}^{(2)}$ is compactly imbedded in $H_{\beta}^{(1)}$, and $B_{2}, B_{1}$ are absorbing balls in $H_{\beta}^{(2)}$, $H_{\beta}^{(1)}$, respectively.

Following [6] we also call $\mathcal{A}_{2, \beta}$ the universal attractor of $S(t)$ in $H_{\beta}^{(2)}$. In order to discuss the existence of a universal attractor in $H_{\beta}^{(1)}$, we first prove the following lemma.

LEMma 5.2. For every $t \geq 0$, the mapping $S(t)$ is continuous on bounded sets of $H_{\beta}^{(1)}$ for the topology of the norm in $L^{2} \times L^{2} \times L^{2}$.

Proof. Let $\left(u_{0 i}, v_{0 i}, \theta_{0 i}\right) \in H_{\beta}^{(1)}(i=1,2),\left\|\left(u_{0 i}, v_{0 i}, \theta_{0 i}\right)\right\|_{H^{1}} \leq R(i=1,2),\left(u_{i}, v_{i}, \theta_{i}\right)$ $=S(t)\left(u_{0 i}, v_{0 i}, \theta_{0 i}\right)$, and $(u, v, \theta)=\left(u_{1}, v_{1}, \theta_{1}\right)-\left(u_{2}, v_{2}, \theta_{2}\right)$. Subtracting the corresponding equations (1.1)-(1.3) satisfied by $\left(u_{1}, v_{1}, \theta_{1}\right)$ and $\left(u_{2}, v_{2}, \theta_{2}\right)$, then multiplying the resulting equations by $u, v, \theta$, respectively, adding together and integrating over $[0,1]$, we get

$$
\begin{aligned}
\frac{1}{2} \frac{d}{d t}\left(\|u\|^{2}+\|v\|^{2}+\|\theta\|^{2}\right)+\int_{0}^{1}\left(\frac{v_{x}^{2}}{u_{1}}+\frac{\theta_{x}^{2}}{u_{1}}\right) d x \\
=\int_{0}^{1}\left(\left(\frac{v_{2 x}+\theta_{2}}{u_{1} u_{2}}-1\right) u v_{x}+\left(\frac{v_{1 x}+v_{2 x}-1-\theta_{2}}{u_{1}} v_{x} \theta\right)\right. \\
\left.+\left(\frac{\theta_{2} v_{2 x}-v_{2 x}^{2}}{u_{1} u_{2}} u \theta\right)+\left(\frac{\theta_{2 x}}{u_{1} u_{2}}-\frac{v_{1 x}}{u_{1}} \theta^{2}\right)\right) d x .
\end{aligned}
$$

By Lemma 3.3, Young's inequality, the imbedding theorem and $\left\|\left(u_{i}, v_{i}, \theta_{i}\right)\right\|_{H^{1}} \leq R$ $(i=1,2)$, we infer from (5.7) that

$$
\frac{d}{d t}\left(\|u\|^{2}+\|v\|^{2}+\|\theta\|^{2}\right)+C_{\beta}\left(\left\|v_{x}\right\|^{2}+\left\|\theta_{x}\right\|^{2}\right) \leq \frac{C_{\beta}}{2}\left(\left\|v_{x}\right\|^{2}+\left\|\theta_{x}\right\|^{2}\right)+C_{R}\left(\|u\|^{2}+\|\theta\|^{2}\right),
$$


where $C_{R}$ is a positive constant depending also on $R$. Then the assertion of this lemma follows from the Gronwall lemma.

Now we can again exactly follow the same argument as in [2] to obtain the existence of a universal attractor in $H_{\beta}^{(1)}$.

THEOREM 5.2. The set

$$
\mathcal { A } _ { 1 , \beta } = \bigcap _ { s \geq 0 } \longdiv { \bigcup _ { t \geq s } S ( t ) B _ { 1 } },
$$

where the closures are taken with respect to the weak topology of $H^{(1)}$, is the universal attractor in $H_{\beta}^{(1)}$.

REMARK 5.2. Since $\mathcal{A}_{2, \beta}$ is bounded in $H^{(2)}$, it is bounded in $H^{(1)}$ and by the invariance property (5.5), we have

$$
\mathcal{A}_{2, \beta} \subseteq \mathcal{A}_{1, \beta}
$$

On the contrary, if we knew that $\mathcal{A}_{1, \beta}$ is bounded in $H^{(2)}$, then the opposite inclusion would hold.

Acknowledgments. Songmu Zheng is partially supported by the grant No. 19831060 from NSF of China, the grant No. 96024603 from the State Education Commission of China, and also by the Laboratory of Mathematics for Nonlinear Sciences in Fudan University. Yuming Qin is partially supported by the Henan Province Science Foundation of China No. 994051100 and also by the Laboratory of Mathematics for Nonlinear Sciences at Fudan University. Qin is currently visiting LNCC/CNPq in Brazil as a visiting researcher supported by a grant from $\mathrm{CNPq}$ in Brazil.

\section{REFERENCES}

[1] G. Chen, Global solutions to the compressible Navier-Stokes equations for a reacting mixture, SIAM J. Math. Anal. 3, 609-634 (1992)

[2] J. M. Ghidaglia, Finite dimensional behaviour for weakly damped driven Schrödinger equations, Ann. Inst. Henri Poincaré 5, 365-405 (1988)

[3] J. K. Hale, Asymptotic behaviour of dissipative systems, Mathematical Surveys and Monographs, Number 25, American Mathematical Society, Providence, Rhode Island, 1988

[4] J. K. Hale and A. Perissinotto, Jr., Global attractor and convergence for one-dimensional semilinear thermoelasticity, Dynamic Systems and Applications 2, 1-9 (1993)

[5] S. Jiang, On the asymptotic behavior of the motion of a viscous, heat-conducting, one-dimensional real gas, Math. Z. 216, 317-336 (1994)

[6] S. Jiang, Large-time behaviour of solutions to the equations of a viscous polytropic ideal gas, Annali di Mat. Pura Appl. 175, 253-275 (1998)

[7] B. Kawohl, Global existence of large solutions to initial boundary value problems for a viscous heat-conducting, one-dimensional real gas, J. Differential Equations 58, 76-103 (1985)

[8] A. V. Kazhikhov and V. V. Shelukhin, Unique global solution with respect to time of initial boundary value problems for one-dimensional equations of a viscous gas, J. Appl. Math. Mech. 41, 273-282 (1977)

[9] T. Nagasawa, On the one-dimensional motion of the polytropic ideal gas non-fixed on the boundary, J. Differential Equations 65, 49-67 (1986)

[10] M. Okada and S. Kawashima, On the equation of one-dimensional motion of compressible viscous fluids, J. Math. Kyoto Univ. 23, 55-71 (1983) 
[11] Y. Qin, Global existence and asymptotic behaviour for the solution to nonlinear one-dimensional, heat-conductive, viscous real gas, Chinese Ann. Math. 20A, 343-354 (1999) (in Chinese)

[12] Y. Qin, Global existence and asymptotic behaviour of solutions to nonlinear hyperbolic-parabolic coupled systems with arbitrary initial data, Ph.D. Thesis, Fudan University, 1998

[13] R. Racke and S. Zheng, Global existence and asymptotic behaviour in nonlinear thermoviscoelasticity, J. Differential Equations 134, 46-67 (1997)

[14] W. Shen and S. Zheng, Maximal attractor for the coupled Cahn-Hilliard Equations, to appear.

[15] W. Shen, S. Zheng, and P. Zhu, Global existence and asymptotic behaviour of weak solutions to nonlinear thermoviscoelastic system with clamped boundary conditions, Quart. Appl. Math. 57, 93-116 (1999)

[16] J. Sprekels and S. Zheng, Maximal attractor for the system of a Landau-Ginzburg theory for structural phase transitions in shape memory alloys, Physica D 121, 252-262 (1998)

[17] J. Sprekels, S. Zheng, and P. Zhu, Asymptotic behaviour of the solutions to a Landau-Ginzburg system with viscosity for martensitic phase transitions in shape memory alloys, SIAM J. Math. Anal. 29, 69-84 (1998)

[18] R. Temam, Infinite-Dimensional Dynamical Systems in Mechanics and Physics, Appl. Math. Sci., Vol. 68, Springer-Verlag, New York, 1988

[19] S. Zheng, Nonlinear Parabolic Equations and Hyperbolic-Parabolic Coupled Systems, Pitman Series Monographs and Surveys in Pure and Applied Mathematics, Vol. 76, Longman Group Limited, London, 1995 